\title{
Fast Zonal DCT-based image compression for Wireless Camera Sensor Networks
}

\author{
Leila Makkaoui, Vincent Lecuire and Jean-Marie Moureaux \\ Centre de Recherche en Automatique de Nancy (CRAN) \\ Nancy-Université, CNRS \\ Campus Sciences, BP 70239 \\ F-54506 Vandœuvre-les-Nancy Cedex, FRANCE \\ e-mail: \{leila.makkaoui, vincent.lecuire, jean-marie.moureaux\}@cran.uhp-nancy.fr
}

\begin{abstract}
This paper deals with image compression over Wireless Camera Sensor Networks (WCSNs) in order to decrease the energy consumption of sensors and thus to maintain a long network lifetime. As the radio tranceiver is the most power greedy components of sensor nodes, it seems natural to consider lossy compression before transmission as the appropriate answer to the problem of energy consumption. However, the limitation of sensor nodes in terms of memory as well as processor speed makes most of the compression algorithms inapplicable. Indeed, the most popular methods such as JPEG or JPEG2000 can yield a higher energy consumption than when transmitting uncompressed images. Here we propose to solve this problem by the design of a fast zonal DCT-based image compression algorithm which allows an efficient tuning of the trade-off between energy consumption and image distortion, as shown by experimental results provided in the paper.
\end{abstract}

Keywords - Image compression, Discrete Cosine Transform, Zonal coding, Wireless sensor networks, Energy conservation.

\section{INTRODUCTION}

The increasing development of Wireless Camera Sensor Networks (WCSNs) allows today a wide variety of applications such as object detection, localization, tracking, and counting. WCSNs have been intensively studied in the literature for the last years especially under the angle of energy consumption for still image transmission as well as for video transmission. Indeed, image-based applications yield the transmission of large data volumes which make the camera nodes as well as the nodes forwarding data towards the sink consuming much more energy than nodes collecting and forwarding scalar data such as, e.g., a 2-byte long temperature. As the radio transceiver is one of the most power greedy components of sensor nodes, compression seems a natural answer to the image transmission problem over a WCSN. Unfortunately, the resource limitation of sensor nodes in terms of memory or processor speed makes most of the compression algorithms inapplicable. For instance, Ferrigno et al. have shown in [7] that the energy consumption involved by popular algorithms such as JPEG, JPEG2000 or SPIHT could be even greater than for the uncompressed image case. As a consequence, special challenges for energy-efficient image compression in WCSNs must be addressed to maintain a long network lifetime.

In this work, we propose an efficient JPEG-like lossy compression scheme which takes into account the DCT energy compaction property that allows to discard high frequency coefficients. This is based on zonal coding, which reduces the number of DCT coefficients to be computed, quantized and encoded, and hence which reduces the computation time at each stage of the whole compression chain. Our scheme relies on both a lifting scheme approach as proposed by Loeffler in [1] and the process of only a small predetermined set of coefficients as proposed in [5], [10]. Combining these two kinds of methods yields the design of a fast zonal DCTbased image compression algorithm. It allows an efficient tuning of the trade-off between energy consumption and image distortion, as shown by experimental results provided in the paper.

Note that if fast DCT algorithms as well as zonal techniques have been intensively studied in the literature [1], [2], [4], [6], [9], [3], [5], [8], there is no work, at our knowledge, that combines both approaches, especially in the WCSNs context. Thus, our contributions are threefold :

- Two zonal DCT techniques have been compared, one using a square shape and the other a triangle shape ;

- Fast Loeffler-based DCT performance is evaluated with respect to both shape and dimension of the DCT selected area ;

- Performance of our proposed compression chain in terms of energy consumption is measured by using an experimental platform which makes reference in the field of WCSNs.

\section{PROPOSAL}

Image compression schemes based on the 2-D 8-point DCT are very popular but this transform is computationally intensive, and hence is energy consuming. There are many works about how to reduce the computation cost of the DCT transform. Most of them are based on the sparse factorizations of the DCT matrix. In the 1-D DCT domain, the Loeffler-Ligtenberg-Moschytz (LLM) algorithm [1], with 11 multiplications and 29 additions, is the most efficient (11 multiplications is the theoretical lower bound). The flow graph of the LLM algorithm is shown in Fig. 1. The 2-D DCT can be obtained by applying first the 1-D DCT over the rows then 
over the resulting columns. Using the LLM algorithm, this requires 176 multiplications and 464 additions. Because multiplications are time-consuming operations, some algorithms such as BinDCT [4], Cordic DCT [6] and Cordic Loeffler DCT [9] approximate multiplications with add and shift operations, at the expense of increasing the image distortion. Among them, the Cordic Loeffler DCT algorithm, with 38 additions and 16 shifts (i.e., 608 additions and 256 shifts for the 2-D DCT), provides the best trade-off between the computational complexity and the image distortion. In the 2-D DCT domain, Feig and Winograd proposed one of the fastest 8-point DCT algorithms, with 94 multiplications and 454 additions [2]. This clearly outperforms the LLM algorithm. Direct 2$\mathrm{D}$ approaches actually have a lower arithmetic complexity than row-column approaches but the processing of large data vectors causes register values to be stored temporarily in memory if the CPU does not have a sufficient number of registers. This can degrade execution speed drastically.

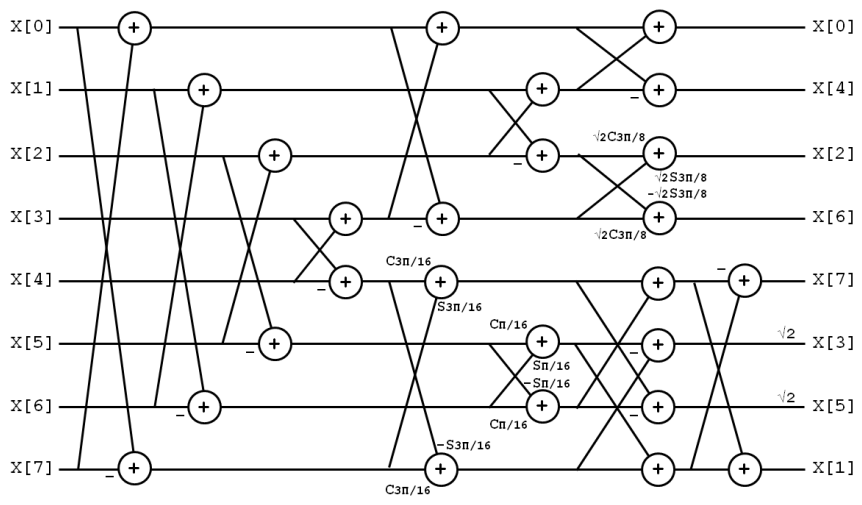

Fig. 1. Flow graph of the LLM 8-point DCT algorithm.

Another approach for reducing the computation cost of the DCT transform is referred to as zonal coding. In zonal coding, only the coefficients within a specified region are encoded and transmitted. In [3] for instance, only a subset of the 64 DCT coefficients have to be computed, quantized and encoded. They are the ones inside the upper-left square portion of size $k$, with $k<8$ (see Fig. 2(a)). The other coefficients are omitted (i.e., they are implicitly set to zero at the decoder side). The smaller the parameter $k$, the lower the computation time in the DCT, quantization, and encoding stages is. Effects of varying parameter $k$ and the quantization level on energy consumption, latency, image quality, and bandwidth are studied in [5]. In [10], the same principle is applied but, here, the concerned shape is delimited by a right-angled triangle included in the previously mentionned portion square (see Fig. 2(b)). As a result, only $\frac{1}{2} k(k+1)$ DCT coefficients are assumed to be important instead of $k^{2}$. Notice that compared to the use of a square pattern, a triangle pattern leads to reduce the computation time in the quantization and encoding stages only. Whatever the pattern, the computation time in the DCT stage remains the same. Indeed, the 2-D 8-point DCT will be achieved in the both cases, by first applying 1-D DCT over the 8 rows then over the resulting $k$ columns.

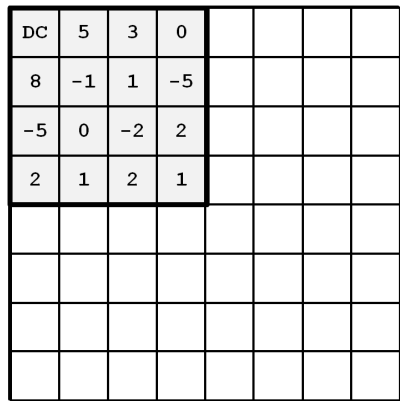

(a) Square pattern

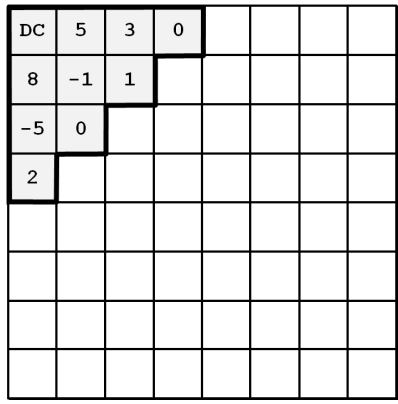

(b) Triangle pattern
Fig. 2. Zonal 2-D 8-point DCT with $k=4$ for different patterns.

Based on the related works about fast DCT algorithms and zonal DCT algorithms, we now propose to combine these two approaches to address the problem of energy consumption in WCSNs, i.e., in resource-constrained, battery-powered wireless cameras. This is referred to as Fast Zonal DCT. We took the LLM algorithm as the starting point for our analysis but the same study could be applied to other fast DCTs. As said before, zonal DCT consists in computing only the most significant DCT coefficients, in other words the low frequency coefficients. The number of coefficients to be computed is defined by the parameter $k$. From the LLM 8-point algorithm, this consists in reducing the number of outputs to $k$. An example is shown in Fig. 3 for the case where $k=4$. With $k=4$, the number of operations is reduced to 9 multiplications and 24 additions. Compared to the original LLM algorithm, the computational savings for the 1-D DCT can seem small, 2 multiplications and 5 additions only. For the 2-D DCT however, the computational savings are clearly high, 68 multiplications and 176 additions, i.e., around 38\% reduction for each kind of operations.

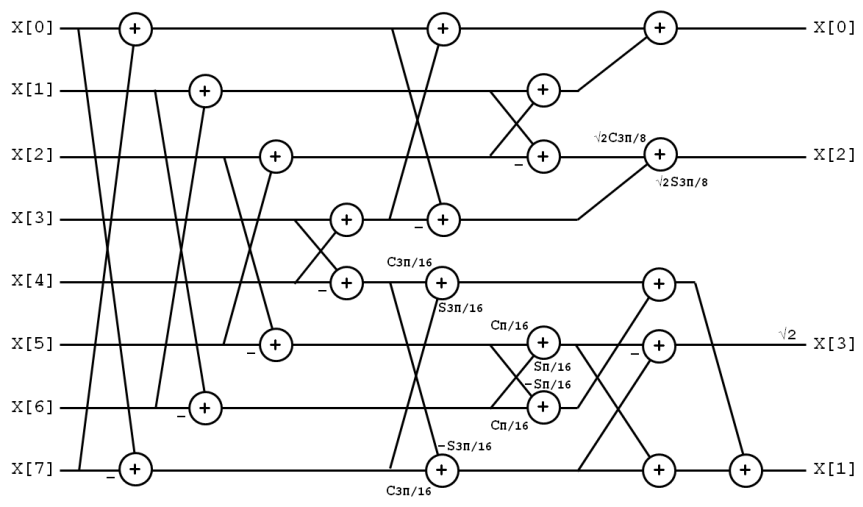

Fig. 3. Zonal coding with the LLM algorithm $(k=4)$.

Table 1 shows the numbers of operations required for differents values of $k$. 


\begin{tabular}{|c||c|c||c|c||}
\hline \multicolumn{1}{|l||}{} & \multicolumn{2}{c||}{ Operations per 1-D DCT } & \multicolumn{2}{c||}{ Operations per 2-D DCT } \\
& multiplications & additions & multiplications & additions \\
\hline LLM (reference) & 11 & 29 & 176 & 464 \\
\hline Zonal LLM (k=7) & 11 & 28 & 165 & 420 \\
\hline Zonal LLM (k=6) & 10 & 26 & 140 & 364 \\
\hline Zonal LLM (k=5) & 9 & 25 & 117 & 325 \\
\hline Zonal LLM (k=4) & 9 & 24 & 108 & 288 \\
\hline Zonal LLM (k=3) & 8 & 23 & 88 & 253 \\
\hline Zonal LLM (k=2) & 6 & 20 & 60 & 200 \\
\hline
\end{tabular}

\section{Performance EVAluation}

The performance of our proposed algorithm has been evaluated considering a JPEG-like compression chain, that is including the quantization and Huffman tables that are recommended in the Annex of the JPEG standard [11]. In the following, we discuss about performance of the two proposed approaches regarding quality of the reconstructed images as well as execution time and energy consumption.

Experimental results in terms of quality are given in Figure 4 which represents image Lena encoded at 0.5 bpp by regular DCT, as well as both square-based and triangle-based zonal DCT for different values of $k$. Obviously, even if the overall visual quality is good, image distortion increases as $k$ decreases for both proposed methods. Furthermore, as we can see in this figure, square and triangle shapes provide approximately the same level of quality (visually speaking and with respect to the PSNR) for large values of $k$ (i.e., 6 and 8 here). On the other hand, for small values of $k$ the square-based method offers a better performance, which is normal since more coefficients are processed. Note that the same tendency has been observed for other values of the coding rate.

To discuss execution time and energy consumption, we have considered the well-known Telos [12], an ultra-low power sensor plateform developed by the University of California, Berkeley. Telos mote consists of a MSP430 16bit microcontroller and a 802.15.4-compliant CC2420 radio transceiver. The microcontroller operates down to $1.8 \mathrm{~V}$ and consumes $3 \mathrm{~mW}$ in active mode (clock speed of $8 \mathrm{MHz}$ ). The radio transceiver has a transmit power of $35 \mathrm{~mW}$ at $0 \mathrm{dBm}$ and provides a data rate of $250 \mathrm{kbps}$. The number of cycles was obtained using the WSim simulator (see http://gforge.inria.fr/projects/wsim/). This simulator provides full instruction set support for the target processor, cycleaccurate and precise simulation of peripherals such as timers or wireless chipsets.

Table 2 gives both execution time and energy consumption related to the proposed square-based and triangle-based approaches for different values of $k$. Using classical JPEG compression, in other words when the 64 DCT coefficients of each block are computed, quantized and coded (see LLM line in table 2), the MSP430 microcontroller requires $43.8 \mathrm{~ms}$ to encode one block, which yields a $132 \mu \mathrm{J}$ energy consumption. As expected, the DCT step is the most computational costly (about $70 \%$ of the total cost). As mentionned previously, the

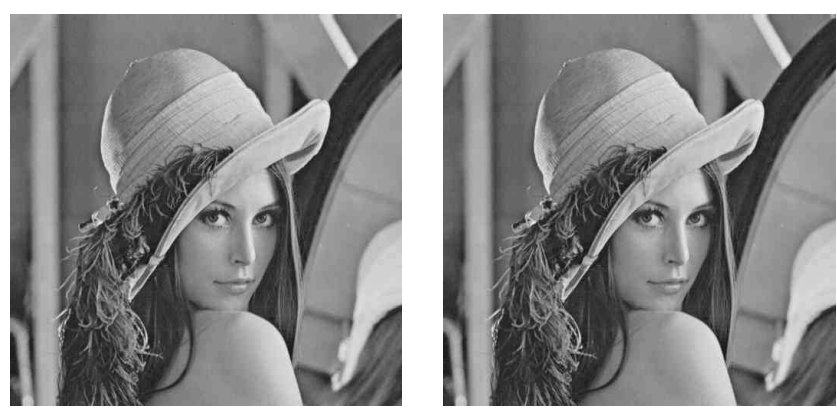

DCT

PSNR $=34.28 \mathrm{~dB}$

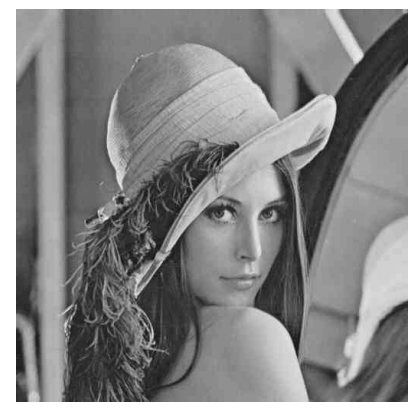

Square-based Zonal DCT $(k=6)$

PSNR $=34.26 \mathrm{~dB}$

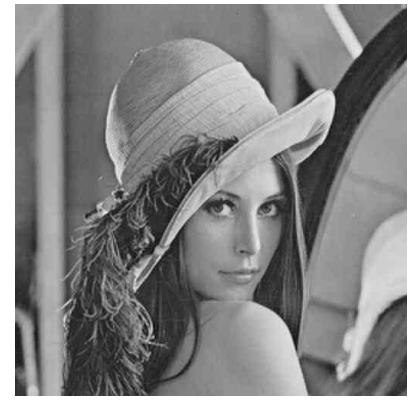

Square-based Zonal DCT $(k=4)$ $\mathrm{PSNR}=33.52 \mathrm{~dB}$

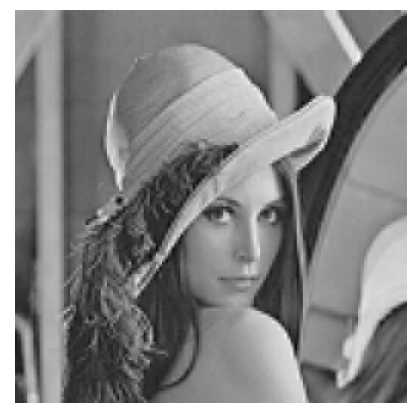

Square-based Zonal DCT ( $k=2)$ PSNR $=28.19 \mathrm{~dB}$
Triangle-based Zonal DCT $(k=8)$ PSNR $=34.28 \mathrm{~dB}$

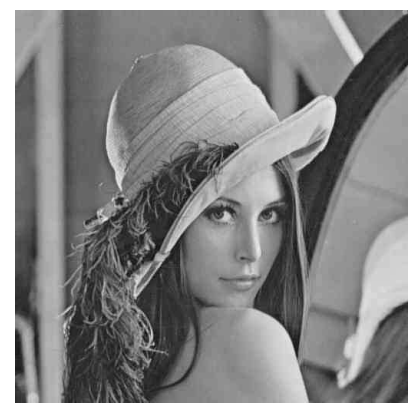

Triangle-based Zonal DCT $(k=6)$ $\mathrm{PSNR}=34.08 \mathrm{~dB}$

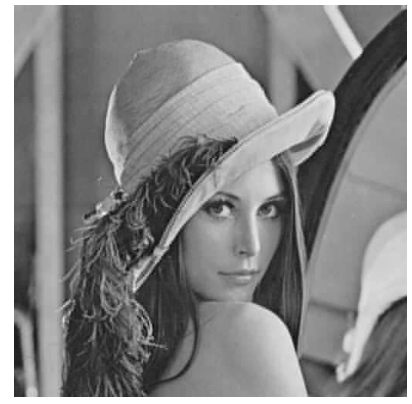

Triangle-based Zonal DCT $(k=4)$ PSNR $=31.79 \mathrm{~dB}$

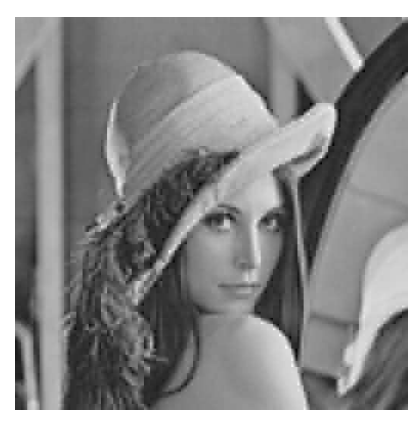

Triangle-based Zonal DCT $(k=2)$ PSNR $=27.35 \mathrm{~dB}$
Fig. 4. Lena image coded at 0.5 bpp by regular DCT, Triangle-based and Square-based zonal DCT for different values of $k$.

DCT cost (in cycles) for a given value of $k$ is the same for both zonal shapes while the quantization cost significantly differs in favor of the triangle shape. Let us consider for example the results related to $k=6$. The square-based approach (see S-6 line) underlines a decreasing cost (in cycles) which 


\begin{tabular}{|c||c|c|c|c||c|c||}
\hline & $\begin{array}{c}\text { DCT } \\
(\text { cycles })\end{array}$ & $\begin{array}{c}\text { Quanti. } \\
(\text { cycles })\end{array}$ & $\begin{array}{c}\text { Coding } \\
(\text { cycles })\end{array}$ & $\begin{array}{c}\text { Total } \\
(\text { cycles })\end{array}$ & $\begin{array}{c}\text { Time } \\
(\mathrm{ms})\end{array}$ & $\begin{array}{c}\text { Energy } \\
(\mu \mathrm{J})\end{array}$ \\
\hline LLM & 246863 & 23980 & 79830 & 350673 & 43.8 & 132 \\
\hline (T-8) & 246863 & 14895 & 77058 & 338816 & 42.4 & 127 \\
\hline (S-6) & 234303 & 14782 & 76926 & 326011 & 40.8 & 122 \\
\hline (T-6) & 234303 & 9898 & 75743 & 319944 & 40.0 & 120 \\
\hline (S-4) & 217455 & 8136 & 75094 & 300685 & 37.6 & 113 \\
\hline (T-4) & 217455 & 6225 & 62667 & 286347 & 35.8 & 107 \\
\hline (S-2) & 169503 & 4190 & 38167 & 211860 & 26.5 & 79 \\
\hline (T-2) & 169503 & 3876 & 33044 & 206423 & 25.8 & 77 \\
\hline
\end{tabular}

yields a reduction of $7.5 \%$ of the energy consumption while the image distortion is similar to the one related to classical JPEG compression ( 34.26 vs. $34.28 \mathrm{~dB}$ ). For the triangle-based approach (see T-6 line), the energy savings is around 9\% at the expense of a slightly higher image distortion (34.08 dB). The maximal gain in energy observed is approximately $42 \%$ per 8-point DCT block (triangle-based approach with $k=2$ ). Thus, combining the advantages of the LLM algorithm and the zonal processing offers significant gains in the lossy image compression context. The choice of the triangle shape instead of the square one should be motivated by the trade-off between energy savings and visual distortion and thus depends of the constraints of the application.

Table 3. Execution time and energy consumption for compression and transmission of a $128 \times 128$ image.

\begin{tabular}{|c||c|c|c|c||c|c||}
\hline \multicolumn{1}{|c||}{} & \multicolumn{2}{c|}{ Compression } & \multicolumn{2}{c||}{ Transmission } & \multicolumn{2}{c||}{ Total } \\
& $\begin{array}{c}\text { Time } \\
(\mathrm{ms})\end{array}$ & $\begin{array}{c}\text { Energy } \\
(\mu \mathrm{J})\end{array}$ & $\begin{array}{c}\text { Time } \\
(\mathrm{ms})\end{array}$ & $\begin{array}{c}\text { Energy } \\
(\mu \mathrm{J})\end{array}$ & $\begin{array}{c}\text { Time } \\
(\mathrm{ms})\end{array}$ & $\begin{array}{c}\text { Energy } \\
(\mu \mathrm{J})\end{array}$ \\
\hline LLM & 11222 & 33665 & 44 & 1548 & 12770 & 35213 \\
\hline R-4 & 9622 & 28866 & 44 & 1548 & 9666 & 30414 \\
\hline T-4 & 9163 & 27489 & 44 & 1548 & 9207 & 29037 \\
\hline R-2 & 6780 & 20339 & 44 & 1548 & 6824 & 21887 \\
\hline T-2 & 6606 & 19817 & 44 & 1548 & 6650 & 21369 \\
\hline NC & - & - & 708 & 24773 & 708 & 24773 \\
\hline
\end{tabular}

Table 3 gives the overall execution time and energy consumption for compression and transmission of a $128 \times 128$ image encoded at $0.5 \mathrm{bpp}$. We considered a protocol overhead of $35 \%$. Classical JPEG compression (see LLM line in table 3) and transmission take $12.8 \mathrm{~s}$ and $35.2 \mathrm{~mJ}$, while the transmission of the original image without compression (see NC line) is faster (less than one second) and less energy greedy $(24.8 \mathrm{~mJ})$. However, decreasing the value of $k$ provides non negligible gains in energy consumption. For example, with $k=4$, these gains equal $14 \%$ and $18 \%$ for square and triangle shapes respectively. Moreover, when taking $k=2$, our scheme outperforms transmission without compression in terms of energy consumption $(21.4 \mathrm{~mJ}$ vs $24.8 \mathrm{~mJ})$. Finally, even if using compression is more time consuming for the source node it allows significant energy savings for all the nodes which forward the data to the sink.

\section{CONCLUSiON}

In this paper we have proposed and evaluated two image compression methods dedicated to transmission over Wireless Camera Sensor Networks (WCSNs) in order to decrease the energy consumption of sensors and thus to maintain a long network lifetime. The two (square and triangle) approaches that are both referred to as fast zonal DCT-based image compression, allow an efficient tuning of the trade-off between energy consumption and image distortion, as shown by experimental results provided in this paper. In a next future our works will be concerned by transposing these results to other fast implementation methods of the literature and to other real sensor plateforms.

\section{REFERENCES}

[1] C. Loeffler, A. Ligtenberg and G. Moschytz. Practical fast 1-D DCT algorithms with 11 multiplications. In IEEE International Conference on Acoustics, Speech, and Signal Processing (ICASSP 1989), Proceedings, 2: 988-991, Glasgow-UK, May 1989.

[2] E. Feig and S. Winograd. Fast algorithms for the discrete cosine transform. IEEE Trans. on Signal Processing, 40(9): 2174-2193, 1992.

[3] J. Bracamonte, M. Ansorge and F. Pellandini. VLSI systems for image compression. A power-consumption/image-resolution trade-off approach. In Conference on Digital Compression Technologies and Systems for Video Communications, Proceedings of the SPIE, 2952: 591-596, BerlinGermany, October 1996.

[4] J. Liang and T. D. Tran. Fast Multiplierless Approximations of the DCT with the Lifting Scheme. IEEE Trans. on Signal Processing, 49(12): 3032-3044, 2001.

[5] C. N. Taylor and S. Dey. Adaptive Image Compression for Wireless Multimedia Communication. In IEEE International Conference on Communications (ICC 2001), Proceedings, 6: 1925-1929, Helsinki-Finland, June 2001.

[6] H. Jeong, J. Kim and W-K. Cho. Low-Power Multiplierless DCT Architecture Using Image Correlation. IEEE Trans. on Consumer Electronics, 50(1): 262-267, 2004.

[7] L. Ferrigno, S. Marano, V. Paciello and A. Pietrosanto. Balancing Computational and Transmission Power Consumption in Wireless Image Sensor Networks. In IEEE International Conference on Virtual Environments, Human-Computer Interfaces and Measurement Systems (VECIMS 2005), Proceedings, Giardini Naxos-Italy, July 2005.

[8] C-H. Hsieh. A Zonal JPEG. In IEEE International Conference on Information Technology: Coding and Computing (ITCC 2005), Proceedings, 2: 756-757, Las Vegas(NV)-USA, April 2005.

[9] B. Heyne, C. C. Sun, J. Goetze and S. J. Ruan. A Computationally Efficient High-Quality Cordic based DCT. In European Signal Processing Conference (EUSIPCO 2006), Proceedings, Florence-Italy, September 2006.

[10] A. Mammeri, A. Khoumsi, D. Ziou and B. Hadjou. Modeling and Adapting JPEG to the Energy Requirements of VSN. In International Conference on Computer Communications and Networks (ICCCN 2008), Proceedings, 806-811, St. Thomas, Virgin Islands-USA, August 2008.

[11] International Organization for Standardization. ITU-T Recommendation T.81. In ISO/IEC IS 10918-1, http://www.jpeg.org/jpeg/.

[12] J. Polastre, R. Szewczyk and D. Culler. Telos: enabling ultra-low power wireless research. In International Symposium on Information Processing in Sensor Networks (IPSN 2005), Proceedings, Los Angeles(CA)-USA, April 2005. 